\title{
Secure Position-Based Routing for VANETs
}

\author{
Charles Harsch ${ }^{1,2}$, Andreas Festag ${ }^{1}$, Panos Papadimitratos ${ }^{2}$ \\ ${ }^{1}$ NEC Deutschland GmbH, \{harsch|festag $\} @$ netlab.nec.de \\ ${ }^{2}$ EPFL, Switzerland, \{charles.harsch|panos.papadimitratos $\} @$ epfl.ch
}

\begin{abstract}
Vehicular communication (VC) systems have the potential to improve road safety and driving comfort. Nevertheless, securing the operation is a prerequisite for deployment. So far, the security of VC applications has mostly drawn the attention of research efforts, while comprehensive solutions to protect the network operation have not been developed. In this paper, we address this problem: we provide a scheme that secures geographic position-based routing, which has been widely accepted as the appropriate one for VC. Moreover, we focus on the scheme currently chosen and evaluated in the Car2Car Communication Consortium (C2C-CC). We integrate security mechanisms to protect the position-based routing functionality and services (beaconing, multi-hop forwarding, and geo-location discovery), and enhance the network robustness. We propose defense mechanisms, relying both on cryptographic primitives, and plausibility checks mitigating false position injection. Our implementation and initial measurements show that the security overhead is low and the proposed scheme deployable. ${ }^{1}$
\end{abstract}

\section{INTRODUCTION}

In the near future, vehicles will be equipped with wireless communication devices, allowing for vehicle-to-vehicle and vehicle-to-infrastructure communication based on short-range wireless technology (IEEE 802.11-like). These vehicular ad hoc networks (VANETs) enable a new set of applications to improve safety, traffic efficiency and driving comfort. For example, a vehicle can warn other vehicles about traffic accidents or bad road conditions.

The specific conditions and requirements for vehicular communication (frequent topology changes, short connectivity times, and the positioning system GPS) have justified the development of a dedicated routing solution for wireless multihop communication based on geographic positions [1], [2], [3]. Position-based routing (PBR) provides scalable and efficient (unicast) forwarding in large-scale and highly volatile ad hoc networks, in contrast to topology-based ad hoc routing. PBR is currently considered and evaluated by the Car2Car Communication Consortium $(C 2 C-C C) .^{2} \mathrm{PBR}$, described in further detail in Sec. II, basically comprises a location service that maps a given node ID to its current position, geographic unicast communication, and distribution of packets in geographic areas. These services, in addition to single-hop broadcast, enable road safety applications that disseminate safety information either as 'event-driven messages' (e.g. de-centralized

\footnotetext{
${ }^{1}$ C. Harsch and A. Festag acknowledge the support of the German Ministry of Education and Research $(\mathrm{BMB}+\mathrm{F})$ for the project 'NoW - Network on Wheels' under contract number 01AK064F.

${ }^{2}$ The $\mathrm{C} 2 \mathrm{C}-\mathrm{CC}$ is an industry consortium that develops a standard for vehicular communication based on IEEE 802.11 technology. http: / / www car-to-car.org
}

hazard warning) or 'periodically-sent beacons' (e.g. extended electronic break-light, forward collision warning). PBR also provides packet transport for vehicle-to-vehicle and vehicle-toroadside roadside communication for non-safety applications.

It is vital to secure communication in VANETs, otherwise the benefits of those novel networks can turn into a nightmare: an attacker could send falsified information to other nodes or block others from receiving safety messages. Since many periodic safety messages are single-hop broadcasted and treated at application layer, the focus has been on securing the application layer. The draft standard of IEEE P1609.2 [4] can not be applied since it does not consider multi-hop routing on a network layer. However, when the network operation is not secured, an attacker could easily partition the network and make delivery of event-driven safety messages impossible. For example, the attacker could advertise a lot of falsified identity/position pairs to its neighbors, thus forcing them to believe there are many neighbors. It is then highly probable that a packet is forwarded to an non-existent node and lost.

In this paper, we address the security of the network layer operation for wireless multi-hop communication in VANETs. We analyze vulnerabilities and potential attacks against geographic routing, and in particular the considered PBR. Based on the analysis, we design a security scheme that protects the PBR services, such as the exchange of nodes coordinates, multi-hop (unicast and broadcast) communication, and the correctness of the location service.

Previous work has presented design principles [5], [6], attacks [7], [8], and components of a secure VANET architecture [9], [5]. Only a few works consider security of geographic routing: [10], [11], [12] focus on plausibility checks for received location beacons, while [13] proposes a secure grid based location service, secure broadcast authentication for hop-by-hop protection, and a reputation system. Compared to [13], our work targets the highly volatile, large-scale VC environment that would make symmetric key mechanisms and reputation systems hard to implement. Our focus is on the communication and networking, and is orthogonal to the described location service. Also, our work in complementary to [10], [11], [12], since it provides a comprehensive security solution and is tailored to the specific PBR protocol.

The rest of this paper is organized as follows: After explaining the basics of PBR in Sec. II, Sec. III lists the target security objectives. Sec. IV provides an attacker model and potential attacks on PBR. Sec. V presents the proposed security scheme and Sec. VI analyzes the achieved security level. Finally, Sec. VII concludes. 


\section{Position-Based Routing Protocol}

Position-based routing provides multi-hop communication in a wireless ad hoc network. It assumes that every node knows its geographic position, e.g. by GPS, and maintains a location table with ID and geographic position of other nodes as soft state. PBR offers geographic unicast (GeoUnicast), topologically-scoped broadcast (TSB, flooding from source to nodes in n-hop neighborhood), geographically-scoped broadcast (Geobroadcast, packet transport from source to all nodes in a geographic area), and geographically-scoped anycast (same as Geobroadcast, but to one of the nodes in the area). Basically, PBR comprises three core components: beaconing, a location service, and forwarding.

Beaconing: Nodes periodically broadcast short packets with their ID and current geographic position. On reception of a beacon, a node stores the information in its location table.

Location Service: When a node needs to know the position of another node currently not available in its location table, it issues a location query message with the sought node ID, sequence number and hop limit. Neighboring nodes rebroadcast this message until it reaches the sought node (or the hop limit). If the request is not a duplicate, the sought node answers with a location reply message carrying its current position and timestamp. On reception of the location reply, the originating node updates its location table.

Geographic unicast provides packet transport between two nodes via multiple wireless hops. When a node wishes to send a unicast packet, it first determines the destination position (by location table look-up or the location service). Then, it executes a greedy forwarding algorithm, sending the packet to its neighbor with the minimum remaining distance to the destination (most forward within radius strategy [14]). The algorithm is executed at every node along the forwarding path until the packet reaches the destination.

Geographic broadcast distributes data packets by flooding, where nodes re-broadcast the packets if they are located in the geographic area carried in the packet. Also, advanced broadcasting algorithms ensure that so-called 'broadcast storms' are avoided and the overhead by packet duplication is minimized.

A number of mechanisms enhance the basic forwarding schemes: if a forwarder has more up-to-date information in its location-table about the destination, it updates the destination position and timestamp values in the header on the fly. Similarly, if the header holds newer information, the node updates its location table.

PBR defines packet headers with fields for node ID, position and timestamp for a source, sender, and destination, and others. ${ }^{3}$ For Geobroadcast, the header carries a destination area instead of a destination ID. For the header fields we distinguish between immutable and mutable fields. Immutable fields are not altered during the forwarding process, whereas mutable fields can be updated by forwarders (see the exemplary Geobroadcast header fields in Fig. 1).

\footnotetext{
${ }^{3}$ The originator of a message is referred to as source, and the last forwarder as sender.
}

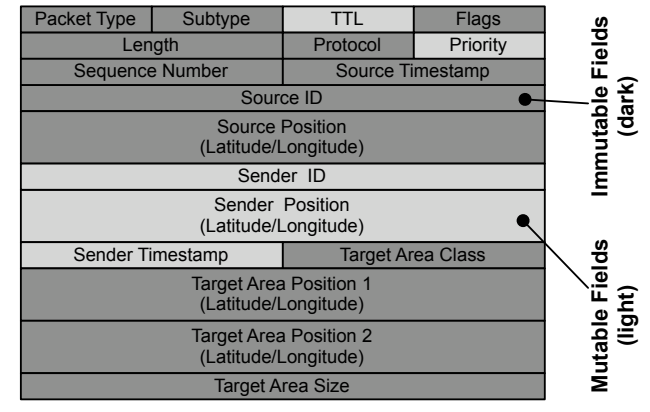

Fig. 1. Example packet type with mutable and immutable fields

\section{Security Objectives}

Since forwarding is based on position information, location information obtained by correct nodes must correspond to the actual node positions, within a degree of accuracy due to the system volatility. More specifically, the location table must include information that is deemed plausible with respect to the reported time and location, and the node's own data.

Plausibility is evaluated based on the received message reporting the location, e.g., beacons or location service replies. In both cases, authentic reporting of position information must be ensured. Nodes are solely responsible for providing their location information and impersonation must be impossible.

Data and control packet forwarding must be loop-free and towards the destination or target area location. ${ }^{4}$ Having packets forwarded across the shortest path towards the destination is not a requirement due to the high network volatility.

The system should be robust against abuse of the positionbased communication services, in particular towards resource depletion. Abuses beyond the PBR functionality (e.g., data link or physical layer jamming) are out of scope.

The above PBR-specific requirements can be related to traditional security requirements. Authentication, and the resultant integrity, and non-repudiation of packets (e.g., beacons and location queries and replies) can be sought as the means to prevent impersonation and other manipulation of location information. Or, facilitate forwarding towards the destination, by preventing alteration of this information in packets. Freshness can be the means to verify plausibility, e.g., by preventing replayed location information. Authorization can assist the robustness, allowing nodes to utilize resources (e.g., initiating geo-broadcasts) according to their assigned roles in the system.

Authenticity, non-repudiation and integrity of data (i.e., payloads) is straightforward as it is a requirement for control packets and fields. Reliability, however, is not a direct requirement at this stage, as communication is not two-way. In the case of broadcast or flooding, reliability is inherent. Redundant forwarding could also assist towards reliability for unicast.

Privacy is not a requirement either, and it is largely orthogonal to our scheme. The selection of a privacy enhancing solution could nonetheless affect the network performance; for the case of frequent pseudonym changes we refer to [15], [16].

\footnotetext{
${ }^{4}$ Except the packet is cached in order to bridge gaps in connectivity.
} 


\section{ATTACKS}

An extensive study on different attacker or adversary models has been presented in [5]. Here we describe the relevant ones to position-based routing in order to guide the design of security countermeasures. Attack trees [17] provide a structured and standardized method to classify attacks on a system. The root represents a general attack further refined in the tree structure using $A N D$ and $O R$ logical connections. The complete attack tree analysis is out-of-scope of this paper, but it is available in [18]. Here we present a simplified version of the sub-tree on denial of service attacks against PBR in Fig. 2. We detail some abuses of PBR next.

\footnotetext{
1. Waste/exhaust resources (OR)

1.1. Waste/exhaust of bandwidth of wireless channels (OR) :

1.2. Create false location table entries in nodes (OR) 1.2.1.Inject messages with false source or sender position 1.2.2.Forward message AND alter source/sender position/ID 1.2.3.Replay previously-heard packet

2. Deter nodes from packet reception (OR)

2.1. Physical jamming

2.2. Drop all data messages (Blackhole Attack)

2.3. Selectively drop data messages (Grayhole Attack)

2.4. Create routing sinkhole AND drop messages (AND)

2.4.1.Inject different beacon messages with false position/ID

2.4.2.Drop data messages

2.5. Create routing loops (OR)

2.5.1. Forward data message on non-greedy route

2.5.2.Inject beacons with false position

2.6. Inject fake reply with wrong position to a location request
} $\vdots$

Fig. 2. Simplified DoS-against-PBR attack sub-tree

- False Position Advertisement: The attacker claims to be at a different position than its actual one, by including this in a packet, e.g., a beacon, or data packet or a location reply.

- Packet Alteration: A forwarder changes, in an unauthorized manner, the original content of the packet header or its payload. Besides manipulating the data payload of the packet, the attacker can poison the location table of subsequent forwarders by altering the source identity or position.

- Geographic Sybil Attack: The attacker advertises multiple IDs and/or positions, to mislead other nodes that high numbers of (non-existent) neighbors exist. This has serious consequences on routing, since other nodes may forward a packet to an non-existing node.

- Packet Dropping: Instead of forwarding a packet, an attacker simply drops it. It can be distinguished between a black-hole attack, where the attacker drops all packets, and a gray-hole attack where the attacker selectively drops packets.

- Replay: The attacker re-injects previously received packets into the network. For example, the attacker can poison a node's location table by replaying beacons.
- Packet Injection: A malicious node creates and transmits bogus packets, injecting location queries or geo-broadcast or unicast packets at high-rates, to waste computation power and bandwidth in a large part of a network.

\section{SECURE PBR}

We design mechanisms safeguarding the functionality of PBR, which relies both on cryptographic primitives and plausibility checks and achieves the stated security objectives. We assume a public key infrastructure $P K I$ with a Certification Authority (CA) that issues public/private key pairs and certificates to vehicles. A certificate contains at least node identifier, lifetime, the node's public key, and its attribute list (e.g., to distinguish between RSUs, public emergency vehicles and regular vehicles) and the signature of the CA.

\section{A. Cryptographic Protection}

Asymmetric cryptography prevents alteration, injection and replay attacks, and the use of digital signatures and certificates allows for immediate communication and provides nonrepudiation.

In case of beacons, signatures can be applied straightforward since there are no intermediate nodes which change PBR header fields. The source signs the whole PBR packet (including potential payload). For all other PBR packet types, mutable fields in the PBR header are updated during the routing process, which implies that a source-generated signature over the whole packet would be invalid at the destination.

Inspired by the view that multi-hop communication is composed of hop-by-hop (neighbor to neighbor) and end-to-end (source to destination) communication, we define a signature scheme in which a packet is protected by two signatures: the source signature, calculated by the source over the immutable fields, and the sender signature, generated by each sending node over the mutable fields (Fig. 3).

On reception of a data packet, a forwarding node $i$ ) verifies both the source and sender signature, ii) updates the mutable field values and generates a new sender signature, iii) replaces the old signature by the new one, and iv) re-forwards the packet. The destination node only verifies both the sender and source signature.

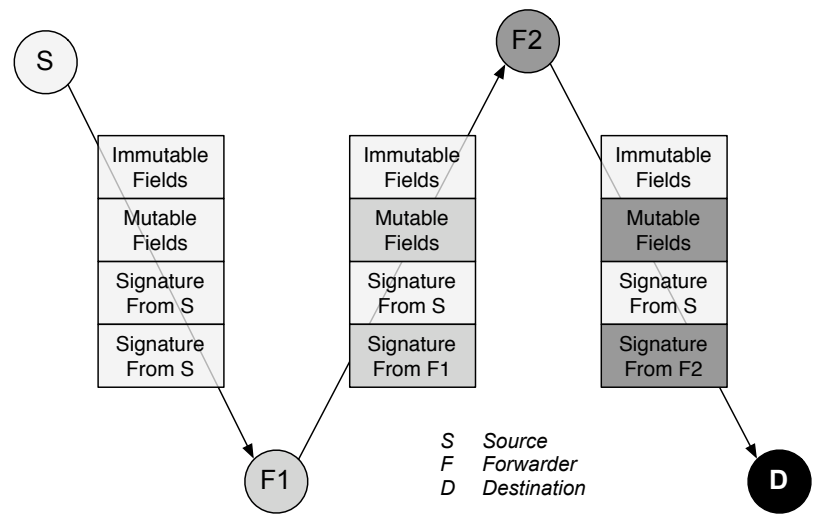

Fig. 3. Hybrid signature scheme: a packet holds a source and a sender signature 


\section{B. Plausibility Checks}

In principle, the plausibility checks compare received data from PBR header fields with some expected value by means of heuristics. When a packet is received, a node first executes a set of plausibility checks and then verifies signature and certificate. Then, nodes update their location table only on PBR header information which is found plausible and the packet header valid, otherwise the packet is dropped. This induces that an attacker advertizing a wrong position is not added to the location table. However, we do not blacklist a node to prevent that an attacker could block a benign node by (instantely) replaying a packet into a region where it would fail the plausibility checks.

Hereafter is a selection of plausibility checks, which extend those presented in [10]:

a) Time: A timestamp is checked for being in a timew window to ensure that it is neither too old (in order to prevent replay attacks), nor it does not lie in the future. In fact, nodes update their location table only if the information of the PBR header is newer. This test ensures that an attacker cannot alter the destination position of a multi-hop packet and set the timestamp to a value in the future; hence, the location tables of all other forwarders are not poisoned.

b) Acceptance Range: Assuming that communication devices have a maximum transmission range $\Delta_{\max }$, no neighbor can be further away than $\Delta_{\max }$.

c) Velocity: The maximum velocity of vehicles is limited by physical laws to $v_{\max }$. Therefore, a claimed position update should be within a predicted space window, calculated around the node's previous position and a radius of $\Delta_{\text {time }} * v_{\max }$ ( $\Delta_{\text {time }}$ is the time between two consecutive position updates).

\section{Robustness Mechanisms}

As the injection of false multi-hop floods or broad- or geocasts wastes resources of a large network part, we propose rate-limiting mechanisms according to the attributes of the sending node. If the rate of such traffic originating a node exceeds a protocol-specific threshold, its packets are not forwarded any further. Digital signatures and unique identification (source time-stamps) of the sender and the transmissions allow this throughout the network. To exert even tighter control, yet maintain effectiveness, we define distinct thresholds for different types of nodes. Furthermore, the description of the transmission (e.g., the geographic area to covered) can correspond to different thresholds. For example, private vehicles can be disallowed to initiate geo-casts beyond a given area size, and allowed to do so at the lowest rate. While RSU units or emergency vehicles can do the same for larger areas, at higher rates, or issue location queries at higher rates.

\section{ANALYSIS}

\section{A. Achieved Security Level}

The use of digital signatures and certificates achieves source authentication, packet integrity and freshness. Likewise, nonrepudiation is achieved, which allows to prosecute misbe- having nodes and might discourage potential attackers from misbehaving.

Since a packet can only be generated and forwarded by authenticated and authorized nodes, the protocol is protected against external attackers. In the following, we analyze the achieved security level against inside attackers.

Spoofing attacks on the GPS signal are out of scope of this paper and we assume that nodes have their correct coordinates ${ }^{5}$. As the PBR header information is secured by digital signatures, no attacker can alter those fields. Furthermore, the location service is protected by the digital signature scheme: an attacker cannot impersonate a sought node nor alter the sought coordinates in the reply packet. Therefore, the location information obtained by correct nodes corresponds to their actual position.

In the assumption that credentials are deployed on a tamperproof device, an insider is in possession of a single certificate and corresponding key pair. As a consequence, he cannot advertise different IDs and the Sybil attack is thwarted.

An attacker is still able to cheat with its position, but its attack possibilities and the resulting impact are drastically reduced by plausibility checks. For example, an insider cannot attract all the traffic by advertizing a position that is farther away than the maximum transmission range or by frequently updating its position so that he is selected as forwarder for a given packet. Furthermore, an attacker cannot misuse the plausibility checks against correct nodes since an implausible packet is simply dropped and no trust level is maintained for nodes.

The impact of packet injection attacks on the overall network performance is reduced by the fact that the sending-rate of regular vehicles is limited.

Packet dropping cannot be prevented. In case of broadcast, a packet is forwarded by a large number of nodes and an attacker which drops packets has a very limited impact on packet delivery (unless he is the only neighbor of the source node). In case of unicast, an insider who is selected as forwarder can stop packet delivery. However, the usage of plausibility checks reduce the risk of selecting an attacker as forwarder, since he cannot attract all the traffic.

\section{B. Comparisons With Alternative Signature Schemes}

In the alternative hop-by-hop $(\mathrm{HbH})$ scheme, the packet is protected by only one signature, calculated by each node on the forwarding chain This scheme assures that only authorized nodes issue and forward packets. However, non-repudiation of the source is not achieved, and an inside attacker can inject or alter packets.

In the end-to-end (E2E) scheme, the source generates a message over the immutable fields. Forwarders are relieved from any cryptographic operation. This scheme allows the destination to verify the message integrity and to authenticate the source node. In addition, non-repudiation of the source is achieved. However, the mutable fields are not protected at all,

\footnotetext{
${ }^{5}$ Future positioning system will be secured, as is the case for the upcoming European satellite navigation system GALILEO
} 
and so an attacker could poison the location table its neighbors by falsifying the sender ID and position.

As last alternative, we design the incremental signature scheme, in which the source signs the whole packet. A forwarder copies the received values of the mutable fields to the end of the packet, updates the mutable values, generates a signature over the packet and appends it to the end. In addition to the achieved security level of $\mathrm{HbH}$, this scheme also enables the destination to authenticate every forwarder (and so build a larger network view), and to track the changes of the mutable fields.

Tab. I presents the number of performed cryptographic operations ${ }^{6}$ for the source, a forwarder and the destination, and provides the total overhead for communication over $\mathrm{n}$ forwarders. We have chosen the hybrid scheme because it offers the best trade-off between achieved security and induced overhead.

\begin{tabular}{|c|c|c|c|c|}
\hline & HBH & E2E & HYBRID & INCREMENTAL \\
\hline \hline SOURCE & 1 & 1 & 2 & 1 \\
FORWARDER & 3 & 0 & 5 & $2 *$ prev_fwd +3 \\
DESTINATION & 2 & 2 & 4 & $2 *(n+1)$ \\
TOTAL & $3 *(n+1)$ & 3 & $6+5 * n$ & $n+1+\sum_{k=1}^{n} 2 * k$ \\
\hline
\end{tabular}

TABLE I

COMPARISON OF NEEDED CRYPTOGRAPHIC OPERATIONS

\section{Initial Measurement Results}

In order to assess the principal deployability of the security solution, we have enhanced our existing prototype for car-tocar communication developed in the project NoW-Network on Wheels ${ }^{7}$ by the proposed security solution and conducted first measurements. The security implementation uses the OpenSSL library, ECDSA with a key size of 160bits for nodes and 224 bits for the CA. The measurements were conducted on a notebook with Intel Pentium M 1,6 GHZ CPU, $256 \mathrm{MB}$ RAM, and Linux operating system. Each measurement was repeated 100 times. Signature generation for a beacon took $\approx 2.9 \mathrm{~ms}$, processing of a signed beacon $\approx 7.7 \mathrm{~ms}$. We regard this initial result as sufficient, but it should (and can) be optimized for beacons received at a high rate in a dense network scenario with many vehicles. We also measured in a lab setup the end-to-end delay of a packet sent over a 4 node multi-hop chain. For a packet with 100 bytes payload, the end-to-end delay was $\approx 77.6 \mathrm{~ms}$, which satisfies the latency constraint (delay $<=100 \mathrm{~ms}$ ) identified by [19] under idealistic conditions.

\section{CONClusion And Future Work}

We have presented a solution to secure a position-based routing protocol for wireless multi-hop communication in vehicular ad hoc networks. Based on an analysis of potential attacks against PBR we have designed a solution to protect

\footnotetext{
${ }^{6}$ Signature verification counts as 2 operations, because the certificate is also verified. It has to be noted that this is only an approximation as the CA signature requires a higher security level than a node signature

${ }^{7}$ http: //www. network-on-wheels.de
}

the PBR services such as the location service and packet forwarding. Our solution combines digital signatures/certificates, plausibility checks, and rate limitation. Digital signatures on a hop-by-hop and end-to-end basis provide authentication, integrity and non-repudiation. Plausibility checks reduce the impact of false positions on the routing operation. Rate limitation reduces the effect of packet injection on a large part of the network.

A main characteristic of the solution is its deployability due to usage of well-established security mechanisms. The integration in our experimental prototype for vehicular communication has shown a low implementation complexity. In a follow-up of this paper we will present a performance evaluation based on measurements in an experimental testbed including further protocol optimzations to decrease the security overhead.

\section{REFERENCES}

[1] B.N. Karp and H.T. Kung. GPSR: Greedy Perimeter Stateless Routing for Wireless Networks. In Proc. MobiCom, Boston, MA, USA, 2000.

[2] H. Füßler, M. Mauve, H. Hartenstein, M. Käsemann, and D. Vollmer. Location-Based Routing for Vehicular Ad-Hoc Networks. In MobiCom, 2002.

[3] A. Festag, H. Füßler, H. Hartenstein, A. Sarma, and R. Schmitz. FleetNet: Bringing Car-to-Car Communication into the Real World. In Proc. ITS World Congress, 2004.

[4] IEEE. Draft Standard for Wireless Access in Vehicular Environments - Security Services for Applications and Management Messages. IEEE P1609.2/D3, 2005.

[5] P. Papadimitratos, V. Gligor, and J.-P. Hubaux. Securing Vehicular Communications - Assumptions, Requirements and Principles. In Proc. ESCAR, 2006

[6] E. Fonseca and A. Festag. A Survey of Existing Approaches for Secure Ad Hoc Routing and Their Applicability to VANETS. Technical report, NEC Network Laboratories, 2006. Available at http://www. network-on-wheels.de.

[7] M. Raya and Hubaux. The Security of Vehicular Ad Hoc Networks. In Proc. SASN, 2005.

[8] A. Aijaz, B. Bochow, F. Dötzer, A. Festag, M. Gerlach, R. Kroh, and T. Leinmüller. Attacks on Inter Vehicle Communication Systems - an Analysis. In Proc. WIT, 2006.

[9] M. Raya, P. Papadimitratos, and J.-P. Hubaux. Securing Vehicular Communications. In IEEE Wireless Communications Magazine, 2006.

[10] T. Leinmüller, C. Maihöfer, E. Schoch, and F. Kargl. Improved Security in Geographic Ad Hoc Routing Through Autonomous Position Verification. In Proc. VANET, 2006.

[11] T. Leinmüller, E. Schoch, F. Kargl, and C. Maihöfer. Influence of Falsified Position Data on Geographic Ad Hoc Routing. In Proc. ESAS, 2005.

[12] T. Leinüller and E. Schoch. Greedy Routing in Highway Scenarios: The Impact of Position Faking Nodes. In Proc. WIT, 2006.

[13] J.-H. Song, V.W.S. Wong, and V. C.M. Leung. A Framework of Secure Location Service for Position-based Ad hoc Routing. In Proc. PEWASUN04, 2004.

[14] H. Takagi and L. Kleinrock. Optimal Transmission Ranges for Randomly Distributed Packet Radio Terminals. IEEE Transactions on Communications, 4(32):246-257, 1984.

[15] E. Schoch, E. Kargl, T. Leinmüller, S. Schlott, and P. Papadimitratos. Impact of Pseudonym Changes on Geographic Routing in VANETs. In Proc. ESAS, 2006.

[16] E. Fonseca, A. Festag, R. Baldessari, and R. Aguiar. Support of Anonymity in VANETs Putting Pseudonymity into Practice. In Proc. WCNC, 2007.

[17] B. Schneier. Attack Trees: Modeling Security Threats, 1999.

[18] Charles Harsch. Secure Position-Based Routing. Master's thesis, Swiss Federal Institute of Technology, Lausanne (EPFL), 2007.

[19] US Vehicle Safety Communications (VSC) Consortium. http://www-nrd.nhtsa.dot.gov/pdf/nrd-12/CAMP 3/ pages/VSCC.htm. 